\title{
THE EFFECT OF ENFLURANE AND FENTANYL ANAESTHESIA ON HUMAN PLATELET AGGREGATION IN VIVO
}

\author{
Alexander W. Gotta, Patty Gould. Colleen A. Sullivan and Paul L. Goldiner
}

\section{ABSTRACT}

In 30 patients undergoing major operations, and anaesthetized with either nitrous oxide. oxygen and enflurane, or nitrous oxide, oxygen and fentanyl, there was no significant alteration in platelet aggregation induced by either adenosine diphosphate or collagen. The absence of any significant effect on platelet function was confirmed by an unchanging thromboelastogram pattern during the study.

Iv 1971 Ueda demonstrated a dose-dependent decrease in platelet aggregation with the in vitro mixing of anaesthetic gases and dog blood.'. Attempts to demonstrate in vivo depression of platelet function in man during anaesthesia and operation have produced equivocal results. ${ }^{2,3,4}$ It was the purpose of this study to assess alterations in platelet aggregation and function in man during the administration of nitrous oxide, oxygen and enflurane anaesthesia and nitrous oxide, oxygen and fentanyl anacsthesia, using the platelet aggregometer and thromboelastograph.

\section{METHODS}

Thirty adult patients undergoing major operations and known not to be taking antiplatelet drugs or anticoagulants were randomly selected to receive either nitrous oxide, oxygen and enflurane, or nitrous oxide. oxygen and fentanyl anaesthesia. Preoperative platelet counts, prothrombin time and activated partial thromboplastin time were normal in all patients. Only one patient in the study had received anti-cancer chemotherapy, but none in the past year. All were evenly matched as to age, sex and type of operation (see Tables 1, 11). The patients were premedicated with morphine sulphate $(5-10 \mathrm{mg}$, pentobarbitone sodium $50-100 \mathrm{mg}$ and glycopyrrolate $0.2-0.4 \mathrm{mg}$ or atropine $0.4-0.6 \mathrm{mg}$. All drugs were administered intramuscularly, ap-

Alexander W. Gotta, M.D. . Director of Research and Education; Patty Gould, R.N., Director of Blood Clotting Laboratory, Department of Anesthesiology and Critical Care Medicine; Colleen A. Sullivan, M.B. Ch.B., Associate Attending Anesthesiologist; Paul L. Goldiner, M.D., Chairman, Department of Anesthesiology. Department of Anesthesiology. Memorial Sloan Kettering Cancer Center, 1275 York Avenue, New York, New York, 10021, U.S.A.

Canad. Anaesth. Soc. J., vol. 27, no. 4, July 1980
TABLE I

Patient Distribution ay Age, Sex

\begin{tabular}{lcccr}
\hline & Male & Female & Age & Mcan \\
\hline Enflurane & 10 & 5 & $38-71$ & 58 \\
Fentanyl & 13 & 2 & $20-67$ & 57 \\
\hline
\end{tabular}

TABLE II

TYPE OF OPERATION

\begin{tabular}{lcc}
\hline & Enflurane & Fentanyl \\
\hline Bowel resection & 0 & 3 \\
Head/Neck & 5 & 1 \\
Urinary tract & 2 & 4 \\
Superficial & 1 & 0 \\
Thoracotomy & $\frac{7}{15}$ & $\frac{7}{15}$ \\
\hline
\end{tabular}

proximately one hour before induction of anaesthesia, the dose being determined by the patient's state of anxiety, height and weight. On arrival in the operating room a 16-gauge central venous pressure line was inserted and all blood specimens were removed through this catheter. In twenty patients (ten fentanyl, ten enflurane) blood was drawn before induction of anaesthesia (control), one hour after start of the operation and in the recovery room within one hour of the end of anaesthesia. Ten of the study patients (five fentanyl, five enflurane) underwent operations which were either preceded by flexible bronchoscopy through the tracheal tube, or by at least one-hatf hour of preoperative preparation under anaesthesia. This allowed time for drawing samples before anaesthesia, during anaesthesia but before the operation, during the operation and in the immediate postoperative period. Thus, if anaesthesia were to induce changes in platelet 319 
function. opposite in character to those induced by operation per se, this would be evident.

The first $10 \mathrm{ml}$ of all blood samples were discarded. Drawn blood was then mixed nine parts to one part $0.11 \mathrm{M}$ sodium citrate. Platelet-rich plasma was prepared by centrifuging whole blood at $270 \times \mathrm{g}$ for ten minutes and removing $2.5 \mathrm{ml}$ of the supernatant. Platelet poor plasma was obtained by further centrifuging the remaining sample at $3000 \times \mathrm{g}$ for twenty minutes. Platelets were maintained at room temperature in capped plastic tubes $1-1.5$ hours before being placed into the aggregometer cuvettes and warmed to $37^{\circ} \mathrm{C}$ for two minutes before testing.

Platelet aggregation was determined using Born's turbidimetric technique which measures alterations in optical density in platelet rich plasma as an index of the number of platelets in suspension. ${ }^{5.6}$ Aggregation decreases the number of platelets in suspension, and thus decreases optical density.

The samples were tested in a PAP-2 aggregometer (Bio-Data Corp., Willow Grove, Pennsylvania, USA), using adenosine diphosphate (ADP) at $2 \times 10^{-5} \mathrm{M}$ concentration, and collagen $0.26 \mathrm{mg} / \mathrm{ml}$ concentration. Aggregation curves, using adjusted platelet rich plasma of 250$300 \mathrm{~mm}^{3}$, were interpreted by measuring the maximum decrease in optical density. Each patient's pre-anaesthetic curves were used as control.

Platelet counts were done on all blood specimens, using a phase microscope.

For thromboelastograph. $y^{7} 0.3 \mathrm{ml}$ of untreated whole blood was pipetted into the rotating cuvette of the thromboelastrograph (Hellige Co., Glenview, Illinois, USA), which had been prewarmed for at least $\mathbf{3 0}$ minutes. The piston was then lowered into the sample, covered with mineral oil and clot formation was measured graphically. The forming clot binds this piston to the rotating cup. Thus the time to beginning clot formation, the clot formation time itself and the tensile strength of the clot can all be measured. Alterations in platelet function (as with any other component of the clotting process), would then be demonstrated as an altered thromboelastogram, when compared to control.

Anaesthesia was induced in all patients with intravenous thiopentone $3-4 \mathrm{mg} \cdot \mathrm{kg}^{-1}$ and tracheal intubation was effected after $1-2 \mathrm{mg} \cdot \mathrm{kg}^{-1}$ of succinylcholine chloride administered intravenously. Pancuronium bromide was used as needed for relaxation during operation and was reversed at the end of the procedure.
Anaesthesia was maintained with 60 per cent nitrous oxide with oxygen, with enflurane $0.5-2.0$ per cent or intermittent intravenous doses of fentanyl as judged necessary. Intravenous fluids consisted of 2.5 per cent dextrose with 0.45 per cent saline $5 \mathrm{ml} \cdot \mathrm{kg}^{-1} /$ hour with whole blood and fresh frozen plasma as needed during the operative procedure.

Two patients received whole blood before the drawing of the intraoperative blood specimen, and the average amount of blood received by these two patients was $300 \mathrm{ml}$. Four patients had received fresh frozen plasma, to an average $150 \mathrm{ml}$.

The data were analyzed using Student's t-test.

\section{RESULTS}

With one exception, all control platelet specimens were aggregated by the adenosine diphosphate solution, and this response was not altered by anaesthesia or operation. Similarly, all control specimens were aggregated by collagen, with no significant alteration in the pattern of aggregation during the study period.

Although the average platelet aggregation response to collagen did decrease slightly after induction of anaesthesia, and before the operation, the change is not statistically significant for either the enflurane or the fentanyl group. Actually, white the mean aggregation did decrease, decreased aggregation occurred in two of five patients receiving enflurane, while in three, aggregation actually increased. Similarly, in the fentanyl group aggregation decreased in two, increased in one and remained unchanged in two patients.

Platelet aggregation increased after beginning the operation, but again the changes were insignificant and unpredictable (See Tables III. IV).

The platelet counts in all patients remained unchanged, except for slight dilutional decreases associated with the administration of large amounts of whole blood or fresh frozen plasma. This slight difference did not alter the study results.

Thromboclastography confirmed the absence of any change in clot formation or the development of clot tensile strength.

\section{Discussion}

Although Ueda's work demonstrated decreased platelet aggregation, it is difficult to as- 
TABLE III

Mean Per Cent Platelet aggregation With Adenosine Diphosphate ( \pm Standard Deviation)

\begin{tabular}{ccccc}
\hline \hline \multicolumn{1}{c}{ Agent } & Preoperative & $\begin{array}{c}\text { Anaesthesia } \\
\text { without operation }\end{array}$ & Intraoperative & Postoperative \\
\hline Fentanyl & $79.6( \pm 9.7)$ & $76( \pm 4.2)$ & $82.5( \pm 9.2)$ & $82.1( \pm 9.6)$ \\
Enflurane & $77.7( \pm 8.8)$ & $77.0( \pm 7.6)$ & $85.3( \pm 9.7)$ & $83.0( \pm 10.9)$ \\
\hline
\end{tabular}

TABLE IV

Mean Per Cent Platelet Aggregation With Collagen ( \pm Standard Deviation)

\begin{tabular}{rcccr}
\hline \hline Agent & Preoperative & $\begin{array}{c}\text { Anaesthesia } \\
\text { without operation }\end{array}$ & Intraoperative & Postoperative \\
\hline Fentanyl & $84.3( \pm 7.5)$ & $76( \pm 10.8)$ & $85.3( \pm 7.9)$ & $87.3( \pm 5.0)$ \\
Enflurane & $83.0( \pm 8.4)$ & $76.4( \pm 5.5)$ & $85.3( \pm 9.1)$ & $85.0( \pm 7.6)$ \\
\hline
\end{tabular}

sess the significance of this in man. In vitro platelet studies are of questionable reliability, Since platelets are notoriously labile. Similarly, species differences among various study animals make it impossible to extrapolate animal data and to consider it applicable to man. ${ }^{8,9}$

Although all patients had cancer, the results of the study are not vitiated, since it has been demonstrated that platelet aggregation induced by adenosine diphosphate is no different in patients with cancer than it is in patients with nonmalignant disease. ${ }^{10}$

O'Brien, Etherington and Jamieson ${ }^{4}$ described a decrease in platelet aggregation in response to both adenosine diphosphate and collagen during thoracotomy. This decreased aggregation was short-lived, returning to normal on the first postoperative day. Although the anaesthetic agents and techniques were not specifically mentioned, it is stated that blood was selected and tested after administration of each anaesthetic agent, including meperidine, thiopentone, curare, neostigmine et al., and that the anaesthetic agents per se apparently had no effect on aggregation. Thus, the decreased platelet aggregation was attributed to release of adenosine diphosphate induced by operation, without further evidence.

Our study, on the contrary, indicates no decrease in platelet aggregation attributable either to anaesthesia or operation.

Platelet aggregation may be thought of as occurring in two stages..$^{9.11}$ Stage I is induced by the addition of adenosine diphosphate, which causes platelets to become sticky, to adhere to each other, to aggregate and precipitate. Stage II may be induced by the addition to platelet rich plasma of collagen, a weaker solution of adenosine diphosphate or epinephrine ${ }^{8,9,11-13}$ causing the release of endogenous adenosine diphosphate from within the platelet, thus increasing the periplatelet concentration of adenosine diphosphate, platelet adhesiveness and aggregation. Our study thus indicates that neither the first stage of platelet aggregation nor the second is altered by enflurane or fentanyl anaesthesia.

Bleeding time determinations were not done because of the lack of correlation between alterations in adenosine diphosphate or collagen induced platelet aggregation and bleeding time. ${ }^{14}$

It has been postulated that a non-specific membrane stablilizing effect of such drugs as chlorpromazine and imipramine may account for their ability to inhibit platelet aggregation, and by extension this membrane stabilizing effect may account for the central nervous system effects of these drugs. ${ }^{15}$ Local anaesthetics have also been shown to inhibit platelet aggregation induced by adenosine diphosphate, ${ }^{16}$ presumably by altering mechanisms of calcium transport and utilization in the nerve. However, we see no indication that general anaesthetics share this membrane effect in platelets and thus suggest that the concept of membrane stabilization cannot logically flow from an effect of anaesthetics on platelet function.

\section{REFERENCES}

1. UEDA, I. The effects of volatile general anesthetics on adenosine diphosphate-induced platelet aggregation. A nesthesiology 34: 405 (1971). 
2. Lichtenfeld, K.M., SChiffer, C.A. \& HelRICH, M. Platelet aggregation during and after gencral anesthesia and surgery. Anesth. Analg. 58: 293 (1979).

3. Kokoros, J.A., Economopoulos. T.C., Alexopoulus, C., Pyrovalakis, J. \& PapayanNIS, A.G. Platelet function tests during major operation for gastro-intestinal cancer. Brit. J. Surg. 64: 147 (1977).

4. O'Brien, J.R., Etherington, M. \& JAMIESON, S. Refractory state of platelet aggregation with major operations. Lancet 2: 741 (1971).

5. BorN, G.V.R. Aggregation of blood platelets by adenosine diphosphate and its reversal. Nature 194: 927 (1962).

6. BoRN, G.V.R. \& Cross, M.J. The aggregation of blood platelets. J. Physiol. 168: 178 (1963).

7. Howlands, W.S., Castro, El B., Fortner, J.B \& Goul.D, P. Hypercoagulability thromboelastographic monitoring during extensive hepatic surgery. Arch. Surg. 108: 605 (1974).

8. Mitchell, J.R.A. \& ShaRP, A.A. Platelet clumping in vitro. Brit J. Haem. 10: 78 (1964)

9. Constantine. J.W. Aggregation of guinea-pig platelets by adenosine diphosphate. Nature 210: 162 (1966).
10. Davis, R.B., Theologides, A. \& Kennedy, B.J. Comparative studies of blood coagulation and platelet aggregation in paticnts with cancer and nonmalignant diseases. Ann. Int. Med. 71: 67 (1969).

11. MaCMillan, D.C. Secondary clumping effect in human citrated platelet-rich plasma produced by adenosine diphosphate and adrenaline. Nature 211: 140(1966).

12. SPAET, T.H. \& ZUCKER, M.B. Mechanism of piatelet plug formation and role of adenosine diphosphate. Amer. J. Physiol. 206: 1267 (1964).

13. O'Brien, J.R. Platelet stickiness. Ann. Rev. Med. 17: 275(1966).

14. Buchanan, G.R., Martin, V., Levine. P.H., SCOON, K. \& HANDIN, R.I. The effects of "antiplatelet" drugs on bleeding time and platelet aggregation in normal human subjects. Amer J. Clin. Pharm. 68: 355 (1977).

15. Mills, D.C.B. \& RoberTs, G.C.K. Membraneactive drugs and the aggregation of human blood platelets. Nature 213: 35 (1967).

16. Feinstein, M.B., Fiekers, J. \& Fraser, C. An analysis of the mechanism of local anesthetic inhibition of platelet aggregation and secretion. $J$. Pharm. Exp. Therap. 197: 215 (1976).

\section{RÉSUMÉ}

Lors d'interventions majeures subies par 30 patients anesthésiés au protoxyde-oxygène fentanyl ou protoxyde-oxygène enflurane, il $n^{\prime} y$ a pas eu altérations significatives de l'aggrégation plaquettaire induite par l'adénosine diphosphate ou le collagène. L'absence d'effet sur la fonction plaquettaire a été confirmée pendant cette étude par la stabilité du thromboélastogramme. 